\title{
Measurement to radius of Newton's ring fringes using polar coordinate transform
}

Ping An ${ }^{1}$, Fu-zhong Bai ${ }^{*}$ (D), Zhen Liu ${ }^{1 *}$, Xiao-juan Gao ${ }^{1}$ and Xiao-qiang Wang ${ }^{2}$

\begin{abstract}
Background: Newton's ring method is often used to measure many physical parameters. And some measured physical quantity can be extracted by calculating the radius parameter of circular fringes from Newton's ring configuration.

Methods: The paper presents a new measuring method for radius of circular fringes, which includes three main steps, i.e., determination of center coordinates of circular fringes, polar coordinates transformation of circular fringes, and gray projection of the transformed result which along the horizontal direction. Then the radius values of each order ring are calculated.

Results: The simulated results indicate that the measuring accuracy of the radius under the effect of random noise can keep the degree of less than 0.5 pixels.

Conclusions: The proposed method can obtain the radius data of each order closed circular fringes. Also, it has several other advantages, including ability of good anti-noise, sub-pixel accuracy and high reliability, and easy to in-situ use.
\end{abstract}

Keywords: Newton's ring, Polar coordinates transform, Fringes pattern, Gray projection

\section{Background}

The parameter estimation of interference fringe patterns has been widely used in optical metrology, including holographic interferometry, electronic speckle pattern interferometry (ESPI) and fringe projection. Such optical techniques have been applied to measure physical parameters such as curvature radius, displacement, strain, surface profile and refractive index. The information regarding the measured physical quantity is stored in the radius parameter of the captured fringe pattern [1]. Some optical fringes, i.e., elementary fringes that have great importance in optical measurement (e.g., Newton's rings fringe patterns), have a quadratic (i.e., secondorder polynomial) phase. Therefore, the fringes pattern is unequispaced fringe.

In general, Newton's rings method is used to measure physical parameters such as film thickness [2, 3], stain [4], and curvature radius [5] as well. In some application, phase demodulation needed to be done in Newton's ring

\footnotetext{
*Correspondence: fzbaiim@163.com; Iz_water@sina.com

${ }^{1}$ College of Mechanical Engineering, Inner Mongolia University of

Technology, Huhhot 010051, China

Full list of author information is available at the end of the article
}

interference configuration. And the Fringe Center Method (FCM) [6, 7] or the Fourier transform $[8,9]$ are still an important inspection method to extract the character information of the fringes pattern. However, for example, the FCM Manual intervention is introduced to link the processes, such as the fringe patching and the assignment of the fringe orders.

In the measurement of curvature radius based of Newton' ring configuration, the radius of fringes is a key parameter and should be accurately obtained from fringes pattern. In the traditional method, the radius of the fringes is measured by observing the microscope and the scale with the eye. The disadvantage of the method is obvious, i.e., the visual field of microscope is small and hence make the fringe center difficult to observe. Additionally, scale is easy to misread due to the fatigue of human eye. Also, parameters of circular fringes can be retrieved with the Fourier transform via the estimation of the phase and its derivatives [9]. However, the required iterative procedure is a time-consuming approach. And it is error-prone because the procedure requires phase unwrapping and numerical differentiation 
operations [10]. The least squares method [11] is also developed to analyzed the circular fringes and estimate the parameter of optical fringes. However, it requires initial approximations for the fringe parameters to be determined.

With the development of digital image processing technology, it has been applied to the fields requiring non-contact, high speed, automatic processing and large dynamic range $[12,13]$. It is especially suitable for the occasion that the traditional method is difficult to be applied. At present, the image processing technique used in analyzing the circular fringes includes several reprocessing steps, such as noise removal, fringe thinning, fringe patching, assignment of the fringe orders and so on [14-16]. For the Hough transform [17, 18] used to determine the parameters and the orders of circular fringes, the computational mount is heavy and the efficiency is low.

Especially aiming at the measurement of radius of plate-convex lens based on the Newton's ring configuration, the paper propose a new analyzing method of the ring fringes to improve automatic processing technique. Through transforming circular fringes to straight fringes with polar coordinates transform, the method carries out the measurement of radius of each order circular fringes. The principle of polar coordinates transform and the processing algorithm of Newton's ring interference pattern are introduced in the paper. Moreover, the accuracy of the method is analyzed and the experiment are done.

\section{Methods}

\section{Principle of polar coordinate transform}

The task of polar coordinate transform is that an image under the Cartesian coordinate $(\mathrm{x}-\mathrm{y})$ space is transformed to another image under polar coordinate $(\phi-\mathrm{r})$ space. The expression of polar coordinate transform is expressed as [19]

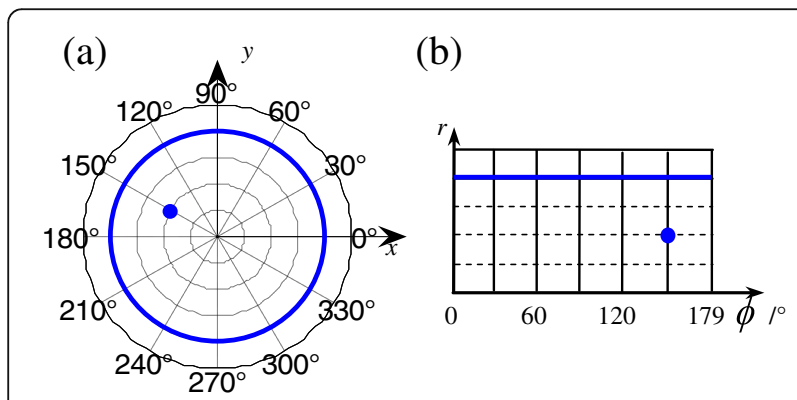

Fig. 1 Schematic diagram of polar coordinate transform: (a) Cartesian coordinate space, (b) polar coordinate space

$$
\left\{\begin{array}{l}
r=\sqrt{x^{2}+y^{2}} \\
\phi=\arctan (y / x)
\end{array} .\right.
$$

The schematic diagram of polar coordinate transform is shown in Fig. 1.

In polar coordinate space, the meaning of $r$ describes the distance of a point $(\mathrm{x}, \mathrm{y})$ to the origin position in Cartesian coordinate space, and $\phi$ discribes the angle of vector and its range is from 0 to $359^{\circ}$. Due to the origin symmetric of polar coordinate transform, the transform needs to be carried out in the range of $0^{\circ}$ to $179^{\circ}$.

According to Eq. (1) and Fig. 1, one point under the Cartesian coordinate space corresponds uniquely to one point under the polar coordinate space. One circle in the Cartesian coordinate space whose center coincides with the origin, will corresponds to one line along $\phi$-axis in the polar coordinate space, and the radius of the circle corresponds to the distance of this line to the origin in polar coordinate space.

\section{Determination of center of circular fringes}

Newton's ring interference fringes is composed of alternating light and dark stripes, and light and dark area are clear, as shown in Fig. 2(a). Through using the Otsu method [20] the fringes image is processed with threshold segmentation, and so a binary image $\mathrm{B}(\mathrm{x}, \mathrm{y})$ can be obtained from the fringes image $f(x, y)$ according to the following expression,

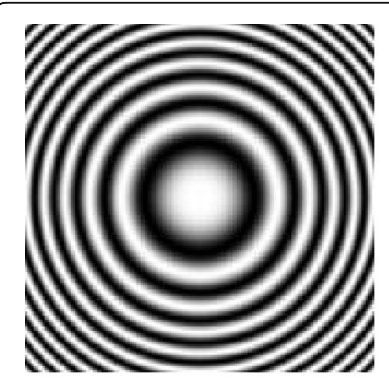

(a)

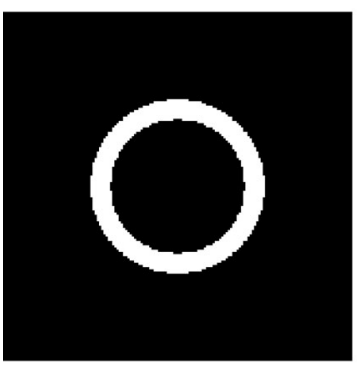

(c)

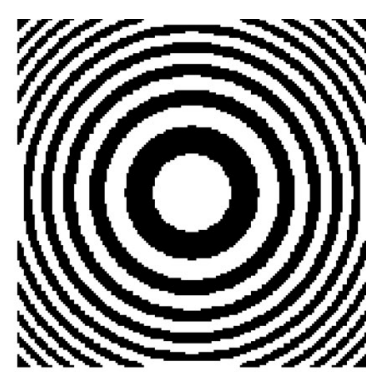

(b)

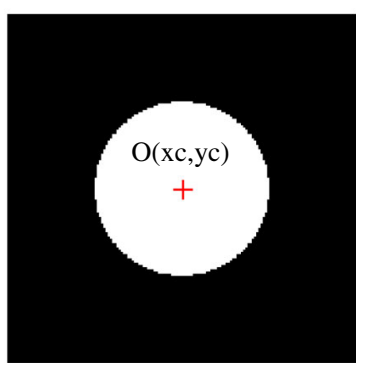

(d)
Fig. 2 Calculation of center ordinates of circular fringes: (a) Newton's ring fringes image, (b) binary image, (c) the first order ring, (d) circular region and center position 


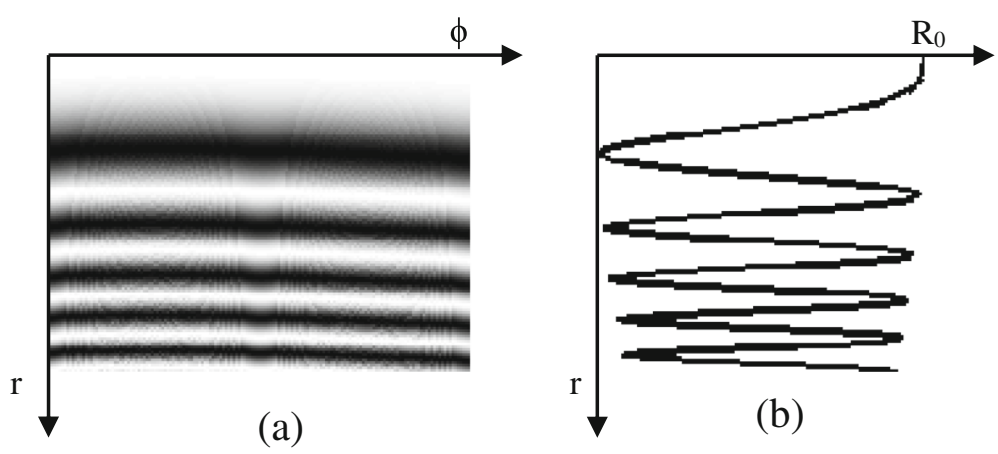

Fig. 3 Polar coordinates transform result and its horizontal gray projection: (a) Polar coordinates transform result of Fig. 2(a), (b) horizontal gray projection of Fig. 3(a)

$$
B(x, y)=\left\{\begin{array}{l}
1, f(x, y)<T \\
0, f(x, y) \geq T
\end{array}\right.
$$

Here, assumed that light fringes are regarded as the target and the radius of light fringes will be calculated, and $\mathrm{T}$ is threshold value. The binary image of Fig. 2(a) is shown in Fig. 2(b).

Through using the connected component labeling algorithm [18] the first order ring from the binary image can be extracted, which is shown in Fig. 2(c). Then, the circular region is filled by the morphological operation, which is shown in Fig. 2(d). Furthermore, the edges of the target region is smoothed by using the opening operation, then the gravity ordinates (xc, yc) of circular region (i.e., the region with white gray-scale pixels) can be calculated according the following equation,

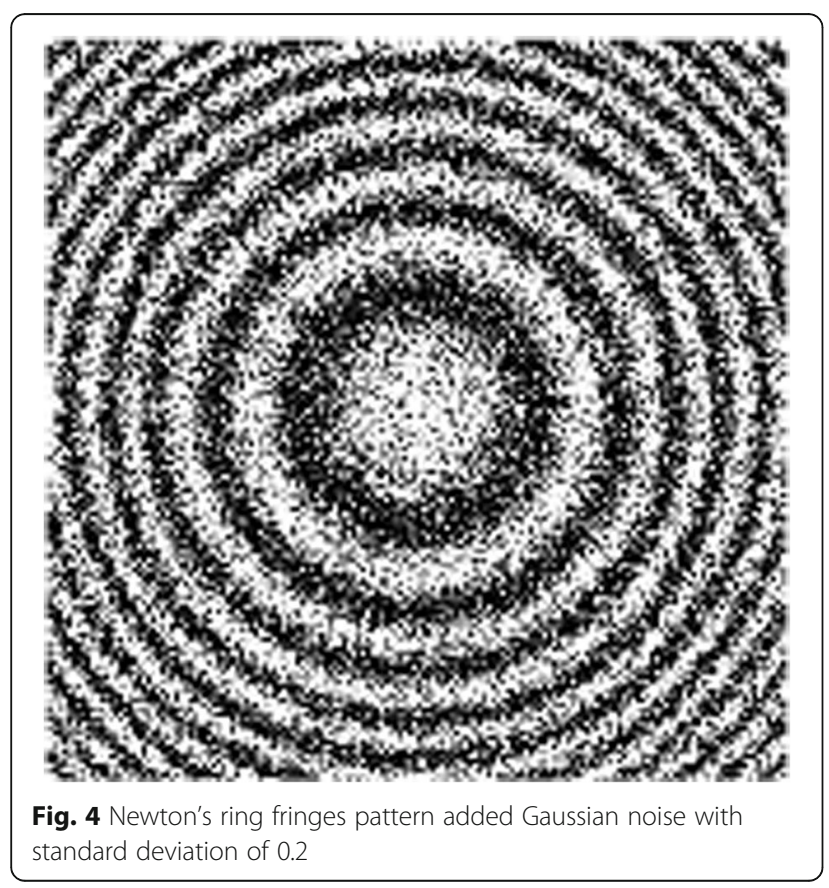

$$
\left\{\begin{array}{l}
x_{c}=\frac{1}{n} \sum x_{i} \\
y_{c}=\frac{1}{n} \sum y_{i}
\end{array},\right.
$$

where, $\mathrm{n}$ is the number of the white pixels as shown in Fig. 2(d). Also, the center of circular fringes is marked in Fig. 2(d).

\section{Calculation of circular fringes radius}

Based on the calculated center the circular fringes are transformed to the polar coordinate space with polar coordinates transform method introduced in Section 2. Therefore, the circular fringes can be transformed to straight fringes. The transformed result of the original image as shown in Fig. 2(a) is shown in Fig. 3(a) and expressed as $p(r, \phi)$.

To calculate the radius of each order ring and eliminate immensely the effect of random noise, the straight fringes as shown in Fig. 3(a) is implemented the horizontal gray projection according to the following equation,

$$
R_{0}(r)=\int p(r, \phi) d \phi
$$

The projection curve of Fig. 3(a) is shown in Fig. 3(b). In the case, the $r$ coordinate value corresponding to each peak position in the projection curve denotes the radius value of each order ring, and hence the method can

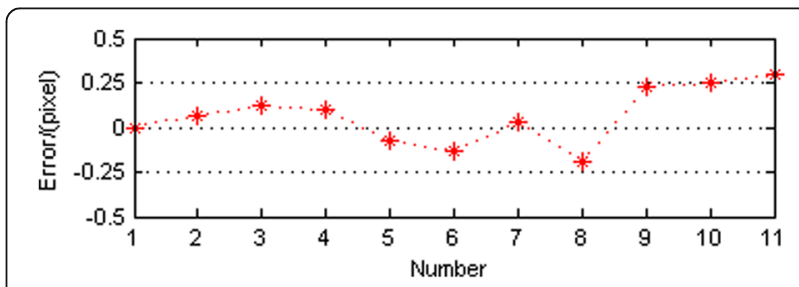

Fig. 5 Center error of the Newton's ring fringes added different noises 


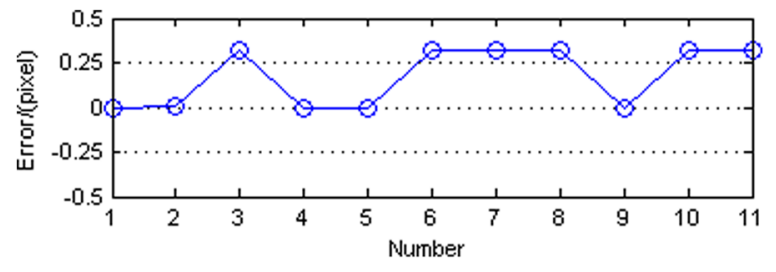

Fig. 6 Radius errors of the Newton's ring fringes added different noises

calculate respectively the radius parameter of each order ring from circular fringes.

\section{Results}

\section{Center positioning accuracy from noise}

The center of circular fringes is one of important parameters to circular fringes, and the center positioning accuracy is affected mainly by the noise. Therefore, it is necessary to analyze the effect of noise on the center positioning accuracy.

Simulated fringes pattern with noise is used to show the effectiveness of the proposed approach as shown in Fig. 4 for an image size of $255 \times 255$ pixels. The center of the simulated image is $(128,128)$. To investigate the effect of random noise on the center positioning, 11 frames of Newton's ring interference fringes containing four closed rings are generated by numerical method, which added to an independent Gaussian white noise with a mean value of zero and standard deviation varying from 0 to 0.2 . The standard deviation of added noise in Fig. 4 is equal to 0.2 .

The center coordinates of each frame of images are calculated by the proposed method introduced in Section 3.1, and the difference between the calculated center and the given center is obtained. The maximum value of the transverse and longitudinal coordinate error is seen as the error of center positioning for each image. The error curve of center positioning is shown in Fig. 5. It can be found from Fig. 5 that the positioning error under the effect of random noise is not larger than 0.5 pixels with the proposed method.

\section{Measuring accuracy of radius}

Similarly, we generate 11 frames of Newton's ring interference fringe patterns, which contain four closed rings, and the size of simulated image is $255 \times 255$ pixels. Then different Gaussian noise with the standard deviation varying from 0 to 0.2 is respectively added to images. For each frame of fringes patterns, the radius values are calculated with the polar coordinates transform algorithm.

According to the horizontal projection curve, the radius of any order closed ring can be obtained. For simple analysis the radius error of the third order ring is seen as the radius measuring error. It can be obtained according to the difference between the measured value and the ideal value of radius. The radius errors of every frame of interference fringes with different random noises are shown in Fig. 6, and the measuring accuracy of the radius can also keep the degree of less than 0.5 pixels.

\section{Experimental result}

To illustrate the performance of the proposed method, this method is applied to an experimental fringes pattern. The Newton's ring experimental setup for recording circular interference fringes is shown in Fig. 7. The $\mathrm{He}-\mathrm{Ne}$ laser with output wavelength of $632.8 \mathrm{~nm}$ is used as experimental light source, and lenses L1 and L2 and spatial filter are used as laser beam expander and collimation. The beam splitter is used to adjust the energy of the reference beam and the object beam. By moving a controlled shifting stage $\mathrm{M}$ driving by the computer, we can record different phase-shifting interferograms. The aperture A1 and A2 are used respectively to control the diameter of laser beam and to filter stray light. The 8-bit

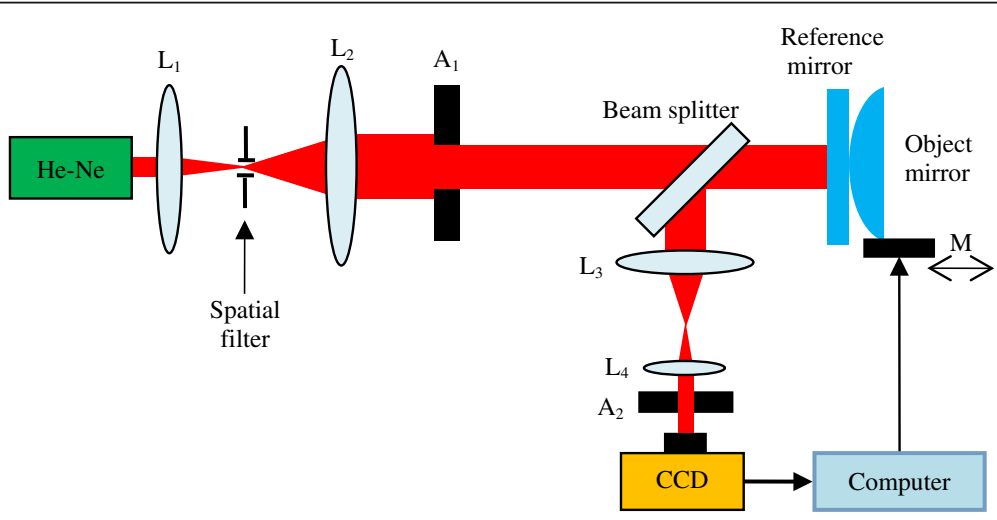

Fig. 7 Newton's ring experimental setup. L1-L4, Lens; A1 and A2, aperture 


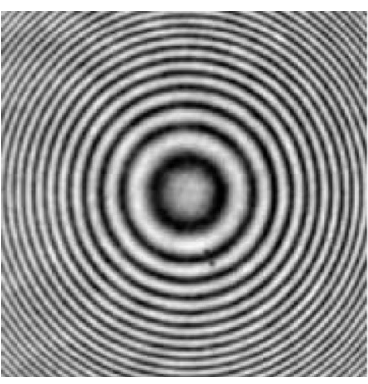

(a)

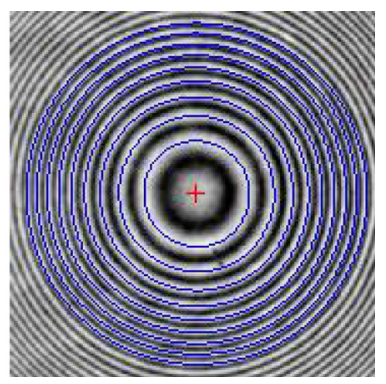

(b)
Fig. 8 An experimental interferogram (a) and the processed results of center position and calculated circles (b)

Basler CCD camera with pixel size of $4.4 \mu \mathrm{m} \times 4.4 \mu \mathrm{m}$ is used to capture the interferograms.

The acquired experimental interferogram with the image size of $454 \times 455$ pixels is shown in Fig. 8(a). The center position and the circular fringes from 1 th to 9 th order as efficient closed-ring are calculated by the proposed method and are plotted in Fig. 8(b). The calculated radius values of each order fringes are shown in Table 1.

According to the measured results, the curvature radius of the lens may be calculated,

$$
R=\frac{r_{m+j}{ }^{2}-r_{n+j}{ }^{2}}{(m-n) \lambda}
$$

where, $\lambda$ is the wavelength of the incident light, $r_{m}$ and $r_{n}$ are the radius of the mth and nth order bright fringes, respectively. If the curvature radius of the lens is known, the wavelength of the incident light can be calculated based on this method and optical setup, and the equation is expressed as

$$
\lambda=\frac{r_{m+j^{2}}-r_{n+j}{ }^{2}}{(m-n) R} .
$$

\section{Conclusions}

The paper proposes a method to analyze the Newton's ring interference fringes. With this method the radius of circular fringes can be determined, and the radius parameter of each order fringes can be obtained. Results of simulation and experiment show that this method hold performance of anti-noise, sub-pixel accuracy and high

Table 1 Calculated radius values of the experimental interferogram

\begin{tabular}{llllllllll}
\hline $\begin{array}{l}\text { Fringes } \\
\text { number }\end{array}$ & 1th & 2th & 3th & 4th & 5th & 6th & 7th & 8th & 9th \\
\hline $\begin{array}{l}\text { Radius/ } \\
\text { pixel }\end{array}$ & 65.5 & 96.5 & 119.8 & 139.5 & 156.5 & 171.8 & 185.8 & 198.8 & 211.5 \\
\hline
\end{tabular}

reliability, and it is convenient to use in in-situ measurement of curvature radius of plate-convex lens. In the practical measurement, we generally use a monochromatic laser output as the incident light. As long as the two order fringes to be measured can be captured by the CCD pixels in the case of fulfilling the sampling theorem, the method is efficient and its measuring accuracy can be ensured. If the incoming light with certain spectral width incidents the Newton's ring configuration, the fringes pattern will show a fall-off of contrast along with increasing the spectral width of the radiation, especially for the more order fringes. In the case, the analysis of this fringe pattern is difficult to many popular methods, but even so the proposed method can still extract its center position and measure the radius values while those order fringes are clear to distinguish and fulfill the sampling theorem. We still believe that the technique provide a new way of image processing in precision measurement and fine interferometry, especially in the analysis of circular fringes pattern.

\section{Acknowledgment \\ This project is supported by the National Natural Science Foundation of China (61108038); Natural Science Foundation of Inner Mongolia of China (2016MS0620, 2015MS0616); Science Foundation of Inner Mongolia University of Technology of China (X201210).}

Authors' contributions

All authors read and approved the final manuscript.

Competing interests

The authors declare that they have no competing interests.

\section{Author details}

${ }^{1}$ College of Mechanical Engineering, Inner Mongolia University of Technology, Huhhot 010051, China. ${ }^{2}$ College of Information Engineering, Inner Mongolia University of Technology, Hohhot 010080, China.

Received: 1 September 2016 Accepted: 30 September 2016 Published online: 11 October 2016

References

1. Rajshekhar, G., Rastogi, P.: Fringe analysis: premise and perspectives. Optics \& Lasers in Engineering 50(8), iii-X (2012)

2. Winston, A.W., Baer, C.A., Allen, L.R.: A simple film thickness gauge utilizing Newton's rings. Vacuum 9(5), 302 (1959)

3. Wahl, K.J., Chromik, R.R., Lee, G.Y.: Quantitative in situ measurement of transfer film thickness by a Newton's rings method. Wear 264(7), 731-736 (2008)

4. Gentle, C.R., Halsall, M.: Measurement of Poisson's ratio using Newton's rings. Opt. Lasers Eng. 3(2), 111-118 (1982)

5. Abdelsalam, D.G., Shaalan, M.S., Eloker, M.M., Kim, D.: Radius of curvature measurement of spherical smooth surfaces by multiple-beam interferometry in reflection. Opt. Lasers Eng. 48(6), 643-649 (2010)

6. Yua, X.L., Yao, Y., Shi, W.J., Sun, Y.X., Chen, D.Y.: Study on an automatic processing technique of the circle interference fringe for fine interferometry. Optik 121(9), 826-830 (2010)

7. Cai, L.Z., Liu, Q., Yang, X.L.: A simple method of contrast enhancement and extremum extraction for interference fringes. Optics \& Laser Technology 35(4), 295-302 (2003)

8. Dobroiu, A., Alexandrescu, A., Apostol, D., Nascov, V., Damian, V.: Centering and profiling algorithm for processing Newton's rings fringe patterns. Opt. Eng. 39(12), 3201-3206 (2000)

9. Nascov, V., Apostol, D., Garoi, F.: Statistical processing of Newton's rings using discrete Fourier analysis. Opt. Eng. 46(2), 28201 (2007) 
10. Kaufmann, G.H., Galizzi, G.E.: Evaluation of a method to determine interferometric phase derivatives. Opt. Lasers Eng. 27(5), 451-465 (1997)

11. Nascov, V., Dobroiu, A., Apostol, D., Damian, V.: Statistical errors on Newton fringe pattern digital processing. Proc. SPIE 5581, 788-796 (2004)

12. Sokkara, T.Z.N., Dessoukya, H.M.E., Shams-Eldinb, M.A., El-Morsy, M.A.: Automatic fringe analysis of two-beam interference patterns for measurement of refractive index and birefringence profiles of fibres. Opt. Lasers Eng. 45(3), 431-441 (2007)

13. Okada, K., Yokoyama, E., Miike, H.: Interference fringe pattern analysis using inverse cosine function. Electronics \& Communications in Japan 90(1), 61-73 (2007)

14. Dias, P.A., Dunkel, T., Fajado, D.A.S., Gallegos, E.L., Denecke, M., Wiedemann, P., Schneider, F.K., Suhr, H.: Image processing for identification and quantification of filamentous bacteria in in situ acquired images. BioMedical Engineering OnLine 15, 64 (2016)

15. Xia, M.L., Wang, L., Lan, Z.X., Chen, H.Z.: High-throughput screening of high Monascus pigment-producing strain based on digital image processing. J. Ind. Microbiol. Biotechnol. 43(4), 451-461 (2016)

16. Li, Y.H., Chen, X.J., Liu, W.J., Yu, Z.H.: Center positioning of circular interference fringe patterns for fine measurement. Optik 125(12), 2796-2799 (2014)

17. Hermann, E., Bleicken, S., Subburaj, Y., García-Sáez, A.J.: Automated analysis of giant unilamellar vesicles using circular Hough transformation. Oxford Journals 30(12), 1747-1754 (2014)

18. Turker, M., Koc-San, D.: Building extraction from high-resolution optical spaceborne images using the integration of support vector machine (SVM) classification, Hough transformation and perceptual grouping. Int. J. Appl. Earth Obs. Geoinf. 34, 58-69 (2015)

19. Lalitha, N.V., Srinivasa Rao, C.H., Jaya Sree, P.V.Y.: An efficient audio watermarking based on SVD and Cartesian-Polar transformation with synchronization. Lecture Notes in Electrical Engineering 372, 365-375 (2015)

20. Zhou, S.B., Shen, A.Q., Li, G.F.: Concrete image segmentation based on multiscale mathematic morphology operators and Otsu method. Advances in Materials Science \& Engineering 2015, 1-11 (2015)

\section{Submit your manuscript to a SpringerOpen ${ }^{\circ}$ journal and benefit from:}

- Convenient online submission

- Rigorous peer review

- Immediate publication on acceptance

- Open access: articles freely available online

- High visibility within the field

- Retaining the copyright to your article 